\title{
The ACS LCID project: overview and first results
}

\author{
Carme Gallart ${ }^{1}$, for the LCID Team $\dagger$ \\ ${ }^{1}$ Instituto de Astrofísica de Canarias, C/ Vía Láctea s/n, 38200 La Laguna, Tenerife, Spain \\ email: carme@iac.es
}

\begin{abstract}
The star formation history ( $\mathrm{SFH}$ ) of Local Group galaxies is a powerful tool for studying their evolution, including chemical enrichment histories and stellar population gradients, which in turn may shed light on the role of reionization or Supernovae feedback in galaxy formation and evolution. In particular, isolated dwarfs are ideal laboratories since their evolution has not been complicated by the vicinity of giant galaxies. In this paper, we present the project Local Cosmology from Isolated Dwarfs (LCID), aimed at deriving detailed SFHs for a sample of Local Group isolated dwarf galaxies. To accomplish this goal we have collected, using the ACS on board the HST, color-magnitude diagrams (CMD) reaching their oldest main sequence turnoff $\left(V \simeq 28\right.$ or $\left.\mathrm{M}_{V} \simeq+3.5\right)$ with good photometric accuracy. Some preliminary results from the CMDs are shown and briefly discussed.
\end{abstract}

Keywords. galaxies: dwarf, stellar content, formation, evolution; Local Group; early universe

\section{Motivation and aims of the project}

The SFH of Local Group (LG) galaxies can be studied very accurately from CMDs reaching the oldest main sequence turnoffs (ideally complemented from spectroscopy from individual stars). It provides key details on the processes of formation and evolution of these galaxies and is, therefore, a complementary path to high-redshift studies of galaxy formation and evolution. The majority of LG galaxies are dwarfs, and dwarf galaxies are also the most numerous type in the Universe. Thus, dwarf galaxies have the potential to provide important information on many key astrophysical processes.

In current structure formation models, dwarf galaxies are the first to collapse, around 1 Gyr after the Big Bang, and thus their oldest populations probe the conditions of the very early Universe. In particular, the details of their early SFHs can provide information on the effects on galaxy formation of the reionization which occurred at high redshift. They also can shed light on the role of other mechanisms, like Supernovae feedback, in removing significant amounts of gas from small galaxy halos.

The MW satellites, the only galaxies which SFHs are known in detail, may however not be good dwarf galaxy representatives for the above purposes. Because of the strong influence of our Galaxy, their evolution is complicated by environmentally related mechanisms like tidal stirring (Mayer et al. 2006) or ram pressure stripping (Blitz \& Robishaw 2000), which are expected to remove gas and strongly affect their SFHs. In addition, the radiation field of the early $\mathrm{MW}$ is expected to dominate over the cosmological UV background (Mayer et al. 2005)

For these reasons, the smallest isolated dwarf galaxies are potentially interesting cosmological probes. Taking advantage of the great sensitivity and spatial resolution of ACS on board the HST, we have undertaken a major project aimed at investigating the SFH of a selected sample of isolated LG dwarf galaxies. Our main goal is to obtain CMDs

$\dagger$ LCID: Local Cosmology from Isolated Dwarfs; http://www.iac.es/project/LCID 


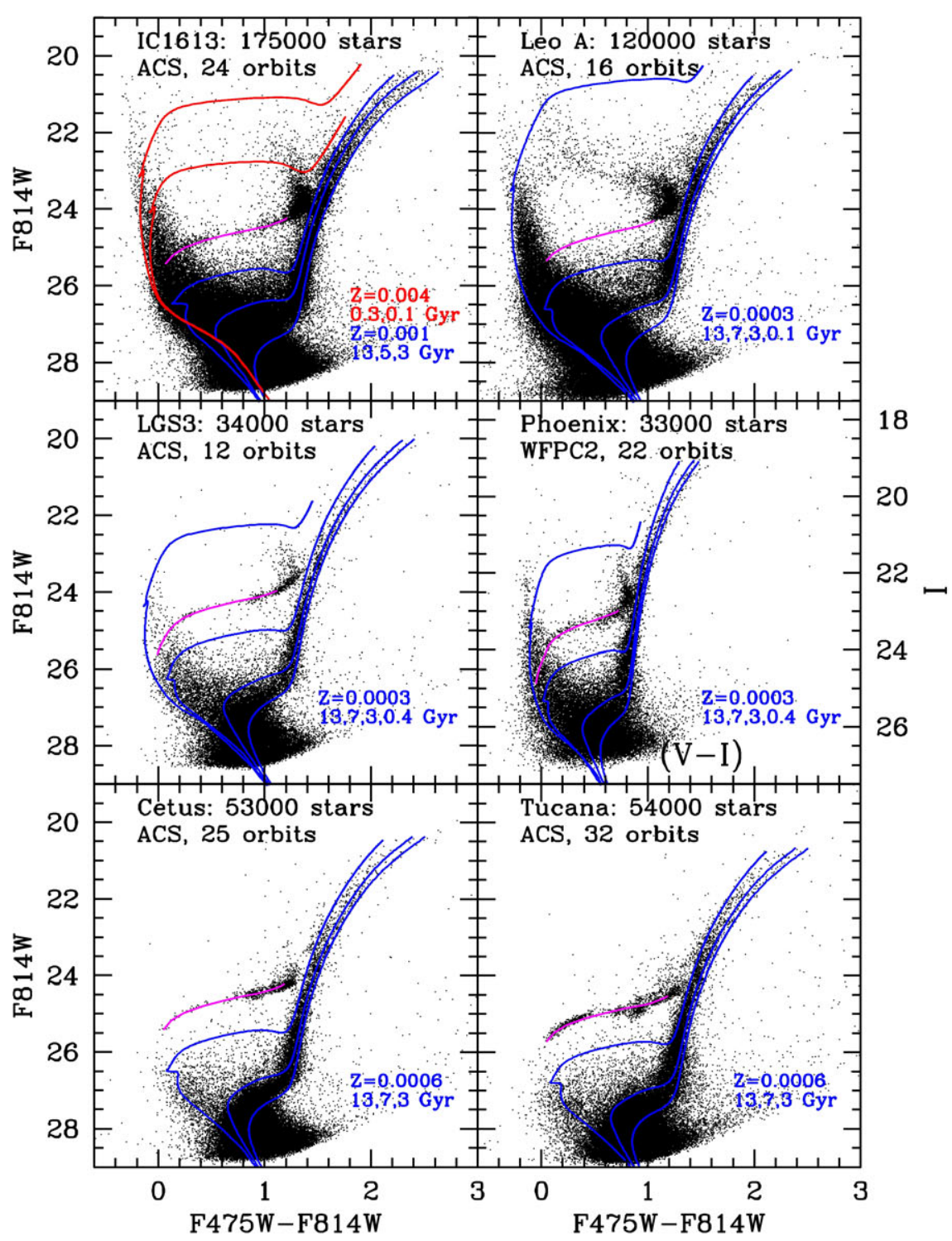

Figure 1. CMDs obtained for the six galaxies in our sample. In order to give a first indication of the range of ages and metallicities present in each galaxy, isochrones from Pietrinferni et al. (2004; overshooting set), with the ages and metallicities indicated in the labels, have been superimposed. The locus of the zero-age horizontal-branch (ZAHB) for $\mathrm{Z}=0.0006$ or $\mathrm{Z}=0.0003$ (depending on the metallicity of the main population) is also shown. Distance moduli of $(m-M)_{0}=$ $24.5,24.5,24.1,23.2,24.5$ and 24.8 and reddenings of $\mathrm{E}(\mathrm{B}-\mathrm{V})=0.04,0.02,0.05,0.02,0.03$ and 0.03 for IC1613, Leo A, LGS3, Phoenix, Cetus and Tucana, respectively, have been adopted. Note the different band combination in the case of Phoenix. Determinations of the SFH of each system are under way through comparison with synthetic CMDs. 
reaching the oldest main-sequence turnoffs $\left(\mathrm{M}_{I} \simeq+3\right.$ in low metallicity systems $)$ with good photometric accuracy, for six isolated dwarf galaxies (two dIrr galaxies: Leo A and IC1613; the two isolated dSph galaxies discovered so far in the Local Group: Cetus and Tucana; and two transition type dIrr/dSph: LGS3 and Phoenix). The data for this project have been obtained through two HST-ACS programs (GO 10505, P.I. Gallart; GO 10590, P.I. Cole) with a total of 113 awarded orbits, and will be supplemented by data from a previous HST-WFPC2 program for Phoenix, (GO 8706, P.I. Aparicio). The main aims of this project are the following:

- To obtain the SFH of each galaxy through comparison of the observed CMD with synthetic CMDs (see below).

- To study the radial gradients in the SFH for the galaxies in the sample, in order to gain insight on the structural evolution within dwarf galaxies.

- To characterize the populations of variable stars in each galaxy, and to understand their relationships with the SFHs and stellar population gradients. This will provide information on the use of variable stars as stellar population tracers.

By comparing the results on isolated dwarfs with those obtained for the MW and M31 satellites, we also expect to shed new light on the effects of environmental mechanisms, which are expected to dominate for the satellite sample. A detailed comparison between Cetus and Tucana, and the dSph satellites will be particularly useful to clarify to what extent these two groups of objects may be related to each other.

Here, we will present some preliminary results regarding the obtained CMDs and the study of the SFH gradients. Other papers in this conference deal with complementary aspects, namely: the data reduction strategy (Monelli), the population of variable stars in Tucana and LGS3 (Bernard), and the first quantitative derivations of the SFH in two of the galaxies: Leo A (Cole) and Phoenix (Hidalgo, Aparicio \& Martínez-Delgado).

\section{The SFH and stellar populations gradients from deep CMDs.}

In this project, we have emphasized the need to obtain CMDs reaching the oldest mainsequence turnoff with good photometric accuracy, in order to obtain reliable and detailed SFHs. In this case, the information can be obtained directly from the main sequence, which is the best understood phase of stellar evolution from the theoretical point of view and is also the one in which the location of stars in the CMD shows the highest sensitivity to age differences. The range of ages and metallicities present can be determined simply through comparison with theoretical isochrones. However, to quantitatively determine the SFH, it is necessary to compare the observed density distribution of stars with that predicted by stellar evolution models (see Gallart, Zoccali, \& Aparicio 2005).

Figure 1 shows the CMDs of all of the galaxies in our sample. Note the variety of SFHs, as evidenced by the comparison with selected isochrones. This is the first time that data of this high quality have been obtained for dwarf galaxies beyond the MW satellite system. The determination of the SFHs, through comparison of observed and synthetic CMDs, is underway using different approaches by different team members (see e.g., Aparicio \& Gallart 2004; Bertelli \& Nasi 2001; Gallart et al. 1999; Skillman et al. 2003; and references therein, for a discussion of the different approaches. See also http://iac-star.iac.es/iacstar/). This will allow us to evaluate systematic differences in the results, and to better understand the errors affecting the SFH determinations. See Cole (this conference, and Cole et al. 2007) and Hidalgo et al. (this conference, and Hidalgo et al. 2007) for the first results on Leo A and Phoenix respectively.

We will also pay special attention to the study of the stellar population gradients. In most cases, the ACS covers a significant fraction of the galaxy, and therefore we will be able to determine a detailed $\mathrm{SFH}$ as a function of galactocentric distance. This will 

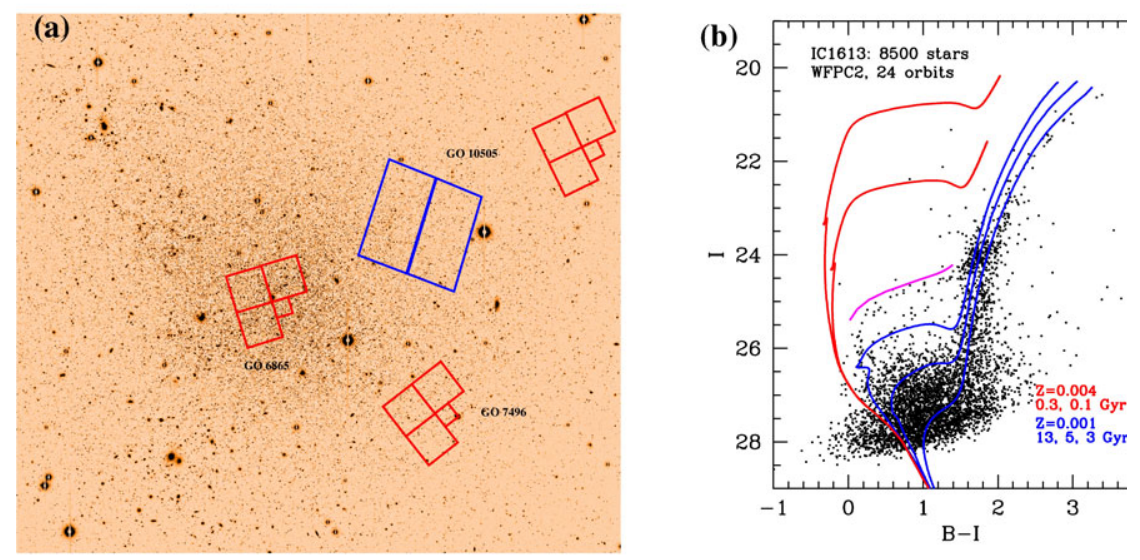

Figure 2. a) IC1613 field from a Subaru archival image. The location of our ACS and WFPC2 fields, and that of other WFPC2 pointings of varying depth is shown. b) CMD of our WFPC2 parallel field, with the same isochrones as in Figure 1 superimposed.

provide key information on the structural evolution of each galaxy over a Hubble time, shedding new light on possible formation mechanisms.

In the case of IC1613, the ACS covers only a small fraction of the galaxy, and we will rely on our WFPC2 parallel field at $\simeq 12^{\prime}$ from the center (plus several archival WFPC2 pointings and ground based data; see Figure 2a) to obtain information on stellar population variations. Figure $2 \mathrm{~b}$ shows the CMD of IC1613 obtained from our WFPC2 exposure. The same isochrones as in Figure 1 have been superimposed. Note the very little presence, if any, of populations younger than $\simeq 2-3$ Gyr. However, a substantial amount of intermediate-age population (as young as 3 Gyr old) exists even in this very peripheral position.

\section{Acknowledgements}

I thank my parents, Juan and Rosa, for coming with me and my little son Daniel to yet another conference, thus allowing me to attend while sharing a special time together. Funding for this project is provided by the IAC (grant P3/94), the Spanish Ministry of Education and Science (grant AYA2004-06343, which includes EU FEDER funds), and NASA (grants GO-10505 and GO-10590 from the STScI).

\section{References}

Aparicio, A. \& Gallart, C. 2004, AJ, 128, 1465

Bertelli, G. \& Nasi, E. 2001, AJ, 121, 1013

Blitz, L. \& Robishaw, T. 2000, ApJ, 541, 675

Cole, A.A. et al. 2007, ApJ Letters, in press

Gallart, C., Freedman, W.L., Aparicio, A., Bertelli, G. \& Chiosi, C. 1999, AJ, 118, 2245

Gallart, C., Zoccali, M. \& Aparicio, A. 2005, ARAA, 43, 387

Hidalgo, S.L., Aparicio, A., Martínez-Delgado, D. 2006, ApJ, submitted

Mayer, L., Mastropietro, C., Wadsley, J., Stadel, J. \& Moore, B. 2006, MNRAS, 369, 1021

Mayer, L. 2005, in IAU Col. 198, Near-field Cosmology with Dwarf Elliptical Galaxies, ed. H. Jerjen \& B. Binggeli, 220

Pietrinferni, A., Cassisi, S., Salaris, M., Castelli, F. 2004, ApJ, 612, 168

Skillman, E. D., Tolstoy, E., Cole, A. A., Dolphin, A. E., Saha, A., Gallagher, J. S., DohmPalmer, R. C. \& Mateo, M. 2003, ApJ, 596, 253 


\section{Discussion}

GEISLER: It is curious that the two strictly dSph galaxies appear to have had very similar SFHs, given the large range in SFHs of the dSphs around the Galaxy.

GALLART: I totally agree, and I am very curious to find out which differences will be detected when we derive the $\mathrm{SFH}$ in detail through comparison with synthetic CMDs. In particular, it will be interesting to see whether we can find differences in the very early $\mathrm{SFH}$ that can be related with clear differences in the HB of the two galaxies.

Rose: If you would allow the mixing length parameter to be maybe 0.1-0.2 different on the giant branch than at the MSTO, could you perhaps reduce the derived age spread and increase the metallicity spread in the galaxy?.

GALLART: One of my collaborators can best answer this question.

CAssisi: Concerning your first question, we use a unique value for the mixing length in the different evolutionary phases. Concerning your second question, I do not think that this would be the case since the same mixing length change would be applied for all chemical compositions. In addition, if we would change the mixing length values with respect to the adopted value (based on the Standard Solar Model calibration), our stellar model for the adopted color- $\mathrm{T}_{\text {eff }}$ relation would not match properly the empirical RGB as they really do!.

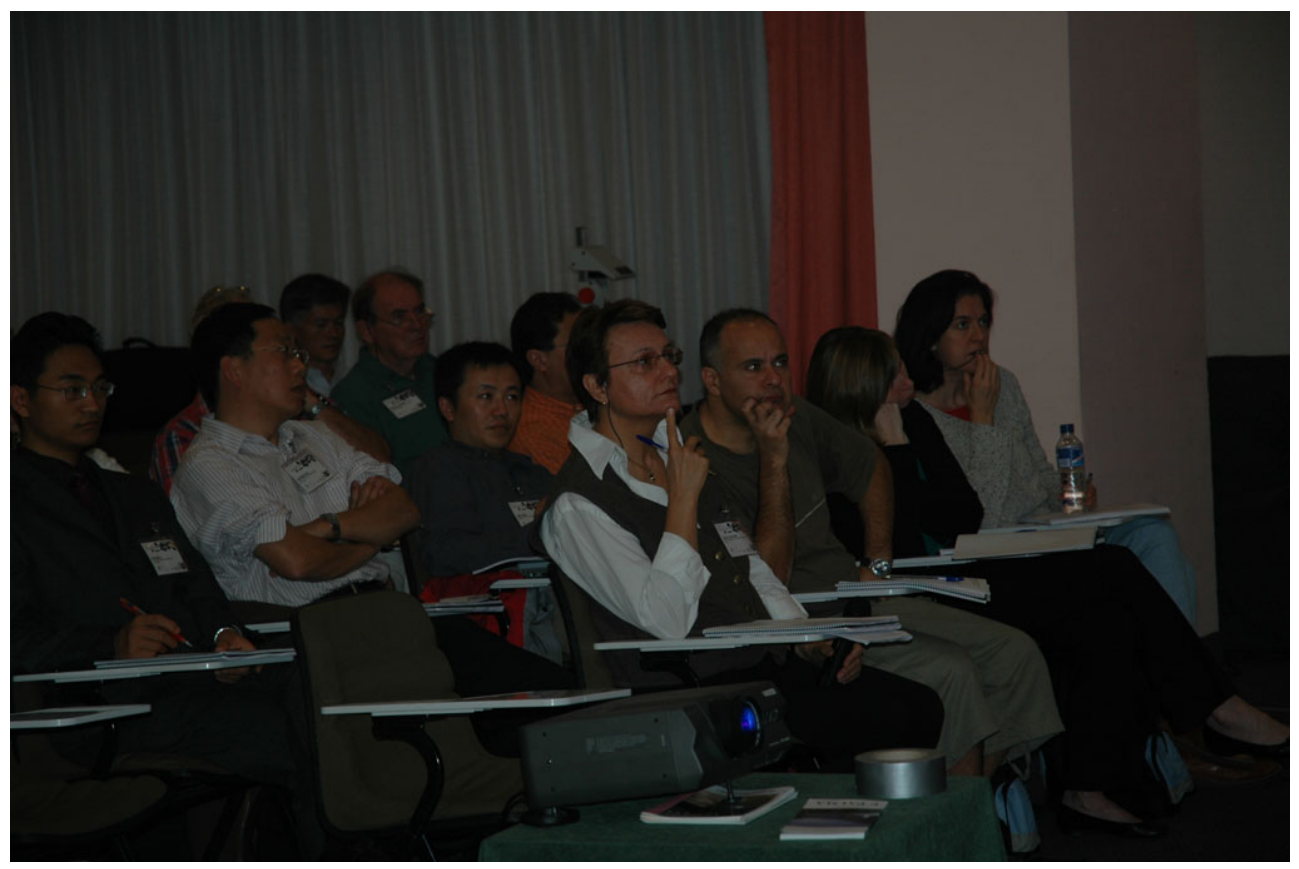

The speaker (on the right of the picture). 